\title{
INTIMIDAD PÚBLICA Y EXPERIENCIA ESTUDIANTIL. REGULACIONES Y SUBVERSIÓN DE LAS NORMAS SEXO- GENÉRICAS EN LA UNIVERSIDAD
}

\author{
Rafael Blanco (Universidad de Buenos Aires- \\ Consejo Nacional de Investigaciones Científicas y Técnicas)* \\ rblanco@sociales.uba.ar \\ Recibido: 29/07/2011 Aceptado: 26/10/2011
}

\begin{abstract}
Resumen
El presente artículo se propone problematizar las modalidades -poco exploradas en relación con otras instituciones educativas- en las que el género y las sexualidades son producidos en las instituciones universitarias. Para ello, se analiza un conjunto heterogéneo de prácticas y discursos presentes en la experiencia estudiantil que opera regulando y desestabilizando las sexualidades en la universidad. Desde una perspectiva etnosociológica, el trabajo se sitúa en las facultades de Ciencias Exactas y Naturales y de Psicología (UBA), y reconstruye los rasgos habituales que adquiere la sociabilidad estudiantil, como lugar problemático de indagación de la normatividad sexo-genérica, en ambos espacios. La investigación se inscribe en un horizonte de indagación más amplio que busca generar conocimiento sobre la universidad pública argentina entendida como espacio de producción de subjetividades y no sólo como un lugar de adquisición de saberes científico- profesionales.
\end{abstract}

\section{Palabras clave}

Universidad - Regulaciones Sociales - Género - Sexualidad - Sociabilidad.

\section{Abstract}

This actual article's aim is to problematize the modalities in wich genre and sexualities are produced in univeritary institutions. For our aim we will analyze an heterogeneous group of practices and discourses that can be found in the pupil's experience wich operations regulates and de-stabilize sexualities at university. From an

* Licenciado en Ciencias de la Comunicación. Universidad de Buenos Aires. Doctorando en Ciencias Sociales. Universidad Nacional de Buenos Aires. Becario del CONICET. 
etnosociologic perspective, this work is focused on the facultys of Ciencias Exactas $y$ Naturales (Natural and Exact Sciences) and Psicología (Psychology) of the Universidad de Buenos Aires (University of Buenos Aires), and re constructs the actual facts acquired by sociability as a problematic space of inquiry about sex-gender normativity -in both spaces. This actual investigation should be inscribed in a bigger horizon whose search is one for knowledge about argentinian public university understood as a space for subjectivity production, and not only as a place in where scientific-proffesional knowledge is acquired.

\section{Key words}

University - Social Regulations - Sexuality - Gender - Normativity.

\section{INTIMIDAD PÚBLICA Y EXPERIENCIA ESTUDIANTIL. REGULACIONES Y SUBVERSIÓN DE LAS NORMAS SEXO- GENÉRICAS EN LA UNIVERSIDAD.}

"Para el conocimiento de la intimidad es más urgente que la determinación de las fechas la localización de nuestra intimidad en los espacios".

Gaston Bachelard, La poética del espacio.

La vida universitaria tiene una relevancia significativa en las biografías de los y las estudiantes no sólo en términos de las expectativas que genera la elección de una carrera, el contacto con nuevos temas, textos, autores y saberes, sino también por el mundo social inédito en el que esa trayectoria educativa se inscribe. En este recorrido, quienes ingresan y transitan la universidad desarrollan una reflexividad particular sobre diversos aspectos de esa experiencia: la formación, la preparación para la vida profesional, los buenos o malos docentes y, de un modo general, el universo social y cultural universitario. Es en función de esta elaboración que articula visiones sobre los pares, la institución y la propia biografía inscripta en una trama reconstruida en parte a partir del artificio de la entrevista, que se busca analizar en este trabajo la dimensión sexuada de la experiencia universitaria. Para ello, el texto se detiene en el análisis de las visiones particulares que los y las estudiantes realizan respecto de prácticas, expresiones e identidades de género y sexualidad heterosexual, lésbica y gay presentes en el espacio universitario. No se analizan en esta ocasión las representaciones y experiencias de vínculos intergeneracionales (por ejemplo, entre docentes y estudiantes) ni un amplio espectro de expresiones e identidades de género y sexualidad no heterosexuales. Este objetivo se inscribe en una horizonte de indagación más amplio que se orienta a generar conocimiento sobre la universidad pública argentina entendida como espacio de producción de subjetividades y no sólo como un lugar de adquisición de saberes científico- profesionales (Carli, 2006). 
Postular la existencia de un ordenamiento implica señalar que -como en todo espacio social- las sexualidades son también producidas (demarcadas, nombradas, diferenciadas, jerarquizadas) y reguladas (es decir, que poseen distintas condiciones de inteligibilidad, posibilidades de expresión, circuitos de socialidad, legitimidades diferenciales) en el espacio universitario. A diferencia de las instituciones de educación primaria o secundaria, en el que prácticas como el hostigamiento o determinadas prescripciones institucionales van produciendo las sexualidades, en la vida universitaria -por diversos factores, como un mayor nivel de autonomía de lo sujetos, un menor peso coercitivo de la institución, la circulación de otros discursos sociales y prácticas- no es posible extrapolar el funcionamiento de estos mismos mecanismos normativos en torno al género y las sexualidades, aunque sea necesario atender a su perdurabilidad aún después del fin del ciclo escolar (como afirma un entrevistado al narrar su llegada a la universidad: "son marcas que en el colegio se rigidizan y quedan ahí"). Por su invisibilidad en los estudios sobre educación como así también en los estudios sobre sexualidades, la universidad aparece como una institución transparente (Blanco, 2010) respecto a la sexualidad: pareciera no haber allí nada específico para analizar con relación al modo en que socialmente se organiza jerárquicamente la legitimidad de las diferentes prácticas e identidades sexuales, como postuló hace años con extrema claridad Gyle Rubin (1989).

Sin embargo, se busca problematizar esta creencia sosteniendo que estas jerarquizaciones se actualizan de un modo particular en el espacio universitario, en tensión con otras regulaciones específicas presentes en la vida cotidiana: las regulaciones del tiempo, de las condiciones espaciales, el establecimiento de lazos de afecto, de compañerismo, entre otros. Es decir, se materializan en los tipos de relaciones -como caracteriza Simmel- "de interacción humanas menores y aparentemente insignificantes según los casos, que al intercalarse entre las configuraciones abarcadoras y, por así decirlo, oficiales, son las que primeramente logran constituir la sociedad tal como la conocemos" (Simmel, 2002: 32).

Con el objetivo de reconstruir algunas características de las regulaciones sexo-genéricas en el espacio universitario, se retoman de los relatos de estudiantes las narrativas principales respecto de la vida cotidiana, atendiendo especialmente a las referencias relativas a los lazos afectivos y sexuales y a las representaciones genéricamente marcadas respecto de los pares. En primer lugar, se busca inscribir el análisis de esta normatividad en el espectro más amplio de la sociabilidad estudiantil. Esto requiere atender a las particularidades que el día-a-día asume en entramados institucionales e intersubjetivos precisos, por lo que el análisis se centra en las narrativas de estudiantes de las facultades de Ciencias Exactas y Naturales ("Exactas") y de Psicología ("Psico") de la Universidad de Buenos Aires a partir de entrevistas realizadas entre los años 2008 y 2010. Desde la perspectiva de los actores (Guber, 1991), y retomando en la exposición expresiones recurrentes en las entrevistas, di- 
ferencio en este apartado dos formas de sociabilidad e itinerarios biográficos diferentes, que acá se denominan como institucionalizados y autogestivos. En segundo lugar, busco ubicar algunas características que adquieren las regulaciones sexo-genéricas desde la perspectiva de los y las estudiantes, a partir de la identificación de las marcas que configuran el espacio universitario como un territorio heteronormativo.

\section{Vida cotidiana y sociabilidad estudiantil}

El inicio de la trayectoria universitaria estudiantil está marcado por una experiencia de ruptura respecto al recorrido biográfico previo. La ruptura se produce no sólo en términos del ingreso a un universo de conocimientos en mayor o menor grado desconocido, sino también por una dinámica de relaciones entre pares e intergeneracional (por ejemplo, en el trato con los docentes) diferente a la de etapas previas (la formación anterior, los lazos con las amistades del barrio) y simultáneas (el trabajo u otras actividades).

Esta posibilidad de poner en relación "mundos de experiencias distintos" propios de la tradición plebeya de la universidad pública argentina (Carli, 2006), es referida en términos de compartir la vida universitaria "con pibes más apretados [económicamente] que otros", "gente de todo el país", con desigualdades de saberes y formación, con diferentes inquietudes, entre otras características. A diferencia de otras instituciones por las que han transitado (universidades privadas, el colegio de procedencia, a algún grupo de interés específico) una referencia habitual acerca del inicio de la vida universitaria refiere a la obligatoriedad de ser "uno más". Ser uno más adquiere el valor de "ser un número" pero también el de pertenecer a una comunidad: encontrarse con "no ser especial", ser "igual que todos". Entre sus dimensiones subjetivas, este inicio habilita el establecimiento de lazos afectivos particulares: amistades, grupos de estudios, noviazgos y relaciones transitorias y ocasionales que, en un tejido institucional e interpersonal específico, articulan el espacio universitario como un espacio de afectos en el que otras reglas organizan una vivencia común.

Como rasgo distintivo en el plano de la sociabilidad, un nuevo marco normativo se abre en esta etapa. El despliegue de una autonomía relativa individual y grupal se produce por el peso que adquiere el plano de las decisiones personales (expresada en una frase recurrente en las entrevistas: "manejar tus tiempos") como así también -como rasgo más significativo- al peso que los lazos entre pares y las expresiones grupales y colectivas adquieren con estatuto de autoridad (lo que supone, en muchos casos desconocer o poner en duda la autoridad de otros: profesores, padres, tradiciones, etcétera). La política estudiantil, por caso, en sus manifestaciones y discursos -de la cartelería a la presencia militante- adquiere una intensidad singular, diferencial respecto a otros espacios institucionales. O la toma, como práctica que visibiliza un acto de apropiación más allá de lo instituido: toma del espacio y ubicación de lugares 
propios; toma de los ámbitos de deliberación e institución de nuevas reglas; toma del espacio destinado para la transmisión del conocimiento y su reconversión a un espacio de fiestas o su apropiación cotidiana para momentos de ocio (en el que, como un entrevistado manifiesta, es posible fumar marihuana sin riesgo de sanción). Es decir, un nuevo espectro de regulaciones de la vida común se realiza a través de reglas específicas en convivencia, tensión, contradicción o complementariedad con las que operan por fuera de esta institución.

Una de las primeras características que regulan el lazo entre pares en la vida cotidiana refiere al tiempo y al espacio que cada facultad instituye para su comunidad. Quienes estudian en Exactas sostienen que es la prescripción de estar cursando "30 o 40 horas por semana" lo que posibilita conocerse. Permanecer mucho tiempo se traduce en una relación estrecha con los compañeros de cursada, con los profesores y ayudantes, que pueden resumirse en la voz de un entrevistado en un "vivir ahí". No es sólo el tiempo de permanencia sino también las condiciones materiales de esa experiencia lo que viabiliza una sociabilidad particular. La posibilidad de estar juntos está dada por la existencia de espacios comunes (la biblioteca, el comedor, los patios internos) y por espacios de cursada que constituyen, como nombra un estudiante de Física, "espacios de obligación" para relacionarse. Esto que es enunciado como una generalidad debe matizarse, sin embargo, en función de posibilidades económicas de cada estudiante. Como sostiene una estudiante de Biología, "chicos que tienen una disponibilidad horaria muy grande y que siempre pueden cursar y armarse grupo. Yo trabajé desde el primer año de la carrera, a mí me costó mucho en ese sentido, de que cursé en cualquier tipo de horario". En Psico, no sólo las cursadas requieren una menor carga horaria (en realidad, se cursa "poco o muy poco" comenta un entrevistado), lo que prescribe una presencia de tiempo parcial -por diferencia al tiempo completo de Exactas- sino también las posibilidades de estar en la facultad son menores por la inexistencia de espacios comunes, a excepción de una serie de pequeños bares, una librería y algunas mesas, todos espacios gestionados por estudiantes en el hall de entrada de una de las tres sedes de esta facultad.

Ya sea como un modo de compartir las largas jornadas en la facultad, ya sea como un modo de sortear las diferencias ante la debilidad institucional (por falta de orientación respecto a los contenidos de las materias, los problemas en las condiciones de cursada, o las astucias administrativas que se imponen como desafío en el inicio de cada cuatrimestre) el establecimiento de amistades constituye un modo particular de atravesar la vida universitaria. Las amistades (que implican modos de relación que trascienden la condición de compañero de cursada para llegar a ser "casi un hermano") proveen orientación mutua en la realización de los recorridos de formación (las amistades como "una guía"), acompañan durante las crisis con la carrera o ante las instancias de evaluaciones (Carli, 2006). 
Si un punto para pensar el modo en que se produce la experiencia estudiantil tiene que ver con el par tiempo parcial/ tiempo completo, que habilita posibilidades diferenciales de establecer lazos con otros, un segundo aspecto tiene que ver con lo que produce en términos de sociabilidad la escala de población de una u otra facultad. De acuerdo con los resultados del censo 2004, el último realizado por esta universidad, la matrícula total de estudiantes de la Universidad asciende a 297.639. Mientras que en Psicología cursan 18.662 estudiantes (el 6,4\%), en Exactas y Naturales Exactas y Naturales 6.041 (el $2,1 \%$ del total de la UBA), es decir, dos tercios menos.

En Exactas, "tenerse visto" constituye una marca relevante. "Somos pocos" es la enunciación recurrente que grafica el ambiente social de esta facultad: "te saludás en el pasillo", "te cruzás con un montón de gente", son expresiones que indican acciones habituales que caracterizan la experiencia estudiantil como un lugar de constante reconocimiento. Esta escala, y su tipo particular de visibilidad y conocimiento del otro, tiene su correlato en un tipo de relación que una estudiante de Computación, califica como "muy íntima" (en la medida en que llega, por ejemplo, a conocer casi la totalidad de los nombres propios de quienes cursan su carrera) y que un entrevistado, de la misma carrera, resume en la figura del consorcio:

Somos muy poquitos y nos conocemos mucho. Es como un consorcio. A ese nivel, porque la gente pasa mucho tiempo acá. Hay por eso mucho chisme. Pueblo chico, infierno grande. [Diego. Entrevista realizada en julio de 2010 por el autor].

En contraste, la masividad en Psico no sólo configura la instancia de formación y el trato con los profesores de un modo particular (en aulas a veces desbordadas, la infraestructura precaria en las tres sedes en las que se cursan las carreras, o incluso la transmisión del docente como "un monólogo" o la escena de la clase, en términos de una entrevistada, como "una misa") sino también en lo relativo al mayor grado de anonimato que articulan los vínculos entre pares. Si en Exactas el lazo con otros está mediado fuertemente por la institución (debido a las posibilidades de estar ahí: en una cátedra con renta, en un laboratorio, con una beca de investigación, pasando el tiempo libre o realizando tareas como divulgador), en Psico, por el contrario, la sociabilidad está marcada por una autogestión de los vínculos en la vida cotidiana. La autogestión, a diferencia de un tipo de experiencia institucionalizada, supone el despliegue de tácticas -en el sentido de cálculos realizados desde una posición débil (De Certeau, 1996)- por parte de los y las estudiantes para generar tiempos en los que compartir la vida en común, espacios de encuentro, de participación, de formación, de inserción laboral, etcétera.

Con todo, tanto los relatos de estudiantes de Exactas como de Psico dan cuenta de formas de sociabilidad articuladas por la participación en una experiencia común en torno a determinados acontecimientos (las cursadas, momen- 
tos de estudio u ocio). Incluso, esa experiencia se materializa en la adscripción a un nosotros que se despliega en múltiples identificaciones: como estudiantes de su facultad, de la Universidad de Buenos Aires, de la Universidad Pública, de la disciplina de estudio o de una comunidad científica, que como rasgo común nombran la inscripción personal en el marco de un colectivo. Sin embargo, las formas que esa sociabilidad adquiere en cada una de las facultades es diferente, y configuran un tipo de lazo producto de una "cronotopía" específica (retomando la expresión de Bajtín que expresa la correlación entre tiempo- espacio- afectos) tal como es experimentada por los y las estudiantes.

Gurvitch (1941), en su estudio clásico acerca de las formas de sociabilidad, identifica dentro del tipo de sociabilidad por unión, propio de las instituciones colectivas, distintas formas posibles (y que denomina masa, comunidad y comunión) y que se diferencian entre sí por la intensidad que adquieren las prácticas, costumbres, rituales cotidianos, tradiciones en cada tipo de vínculo. Esa diferencia puede condensarse, para las formas de sociabilidad en estas dos facultades, en las figuras del consorcio -tomando una categoría nativa- y la gran ciudad.

Las figuras permiten reconocer "algo que ha sido dicho, escuchado, experimentado" (Barthes, 2002: 14) y mediante estas dos nominaciones busco graficar las polaridades (conocimiento del otro/ anonimato; familiaridad/ masividad; espacio propio/ espacio otro) en las que se desarrolla la sociabilidad, en ambos casos limitada, en cada una de las facultades. Si esta contraposición vale para pensar múltiples esferas que articulan la vida estudiantil, vale también para problematizar las regulaciones sexo-genéricas en estas formas de sociabilidad.

Si en los últimos años, por fuera de la institución -especialmente en la ciudad de Buenos Aires- ha habido fenómenos emergentes de visibilización y movilización de prácticas y discursos relativos a la sexualidad que marcan una progresiva intervención en el espacio público (Moreno, 2008), cabe la pregunta respecto a qué sucede con este fenómeno a escala de la universidad que tiene la peculiaridad, en el caso de la UBA, de encontrarse emplazada en la trama urbana. A su vez, su adentro está conformado entre otros actores por estudiantes, en su mayoría jóvenes, que constituyen un componente dinámico de la vida universitaria. No como un colectivo homogéneo, pero sin desatender al componente epocal, algunos trabajos actuales -localizados sobre poblaciones específicas- señalan que las generaciones más jóvenes se socializan en nuevo clima normativo, menos prohibitivo respecto a la sexualidad (Margulis, 2007), o en un creciente marco de modernización, producto de un proceso de secularización y mayor reflexividad en torno a los géneros y sexualidades, no exenta de contradicciones (Jones, 2010). Abordar la normatividad de las sexualidades en el espacio universitario supone identificar los mecanismos particulares a partir de los que se van construyendo los límites inestables, 
cambiantes, sujetos a redefinición pero no por eso inexistentes que regulan los recorridos biográficos.

\section{Escenas de intimidad pública, visibilidad y normatividad de las sexualidades}

Al igual que las amistades, el tópico de las relaciones amorosas y sexuales es reiterado en los relatos de estudiantes: vínculos que se arman durante las horas de estudio y preparación de un trabajo práctico, en las fiestas, en los espacios de militancia, o en el foro virtual, en el que debaten temas relativos a las cursadas, la política pero, de un modo más enfático, en el que prima una dinámica lúdica de intercambio (“¿Qué se debate en el foro? 90\% son boludeces. Desde subir videos de Youtube hasta levantarse mías o chabones. Después si, tenés una sección aparte de política o del estatuto", comenta un estudiante de biología). Este tópico se despliega en un repertorio amplio de relaciones posibles, desde el acontecimiento de "conocer a alguien" hasta entablar un vínculo de noviazgo o inaugurar una vida sexual que, en ocasiones, tiene primacía en su significación respecto al contacto con un determinado universo de conocimientos. "Lo más positivo tuvo que ver con el sexo y no con las clases del CBC", dice un entrevistado de Psicología:

Me impresionó un poco eso y los recuerdos los tengo muy asociados a salir del claustro de lo que es el colegio y a tener una vida sexual un poco más abierta, punto. Más circulación de mujeres que te pueden gustar y que pueden gustar de vos. [Camilo. Entrevista realizada en agosto de 2010 por el autor].

La particularidad del tiempo/espacio compartido en Exactas facilita lo que varios estudiantes denominan "la teoría de la endogamia", que refiere al establecimiento de vínculos afectivos y sexuales con gente de la misma facultad. Comenta una estudiante de Biología, ante la pregunta respecto a si son habituales las "historias" en la facultad, que en [el equipo de la facultad de] voley es algo muy raro porque casi todo el equipo de chicas se puso a salir con todo el equipo de chicos (...) Es más, unos chicos que estaban antes que nosotros, desde hace años, ahora se casaron y tuvieron un chico, así que sí, es normal. [Julieta. Entrevista realizada en enero de 2010 por el autor].

Compañeros "que se terminan casando", amigos del grupo que están de novios "con gente mismo de la facultad", parejas que son "compañeros de laboratorio, que hacen materias juntos", alguna relación "nada trascendente", son algunos de los relatos singulares pero inscriptos en una narrativa común respecto a la habitualidad de los lazos afectivos y sexuales en la facultad y que refieren a diferentes escenas de intimidad pública.

Intimidad pública parecería nombrar -como señala Leonor Arfuch- una contradicción entre los términos. Sin embargo, esta frase marca la tensión entre dos esferas en las que, mientras la primera parecería referir a lo que "más 
intrínsecamente nos constituye y representa", requiere para su reconocimiento el enfrentarse "a su exterioridad, lo que no es ella, su opuesto -o su complementario- , el otro lado del umbral, lo público" (Arfuch, 2005: 239). Esta tensión entre esferas resulta pertinente para problematizar las escenas de noviazgo, levante, seducción, visibles en el espacio (público) de las facultades y los relatos de estudiantes, imbricados en el horizonte más abarcativo de las regulaciones heteronormativas, como "principio organizador del orden de relaciones sociales, política, institucional y culturalmente producido, que hace de la heterosexualidad reproductiva el parámetro desde el cual juzgar (aceptar, condenar) la inmensa variedad de prácticas, identidades y relaciones sexuales, afectivas y amorosas existentes" (Pecheny, 2008: 14).

En Psico, las referencias a este espectro de vínculos son también habituales; sin embargo, presentan otras características. Psicología es caracterizada como una facultad de "mayoría de mujeres", que se completa con otras minorías. Según el Censo de Estudiantes 2004, de los 24052 estudiantes, la cantidad de varones en la carrera de Psicología era de $4280(17,81 \%)$ frente a 19751 $(82,18 \%)$ mujeres (21 sin respuesta). Como un estudiante describe:

Un $80 \%$ de la facultad son mujeres. Un $20 \%$ somos de sexo masculino. Un $10 \%$ somos varones y el resto te diría que algo indefinido. [Juan. Entrevista realizada en octubre de 2009 por el autor].

Indefinido refiere, en palabras del entrevistado, a que en la facultad hay "bastantes homosexuales". Esta creencia circula con diferentes enunciaciones que no pueden ser leídas -como se retoma más adelante- unívocamente como peyorativas ("Si hay un chico en la facultad, o es raro o es puto"; "la facultad se compone de mayoría de mujeres, muchos gays y algunos varones", "los tres hombres que hay [en una clase] habitualmente son putos"). El estudiante-gay es identificable, según los y las estudiantes, por "rasgos femeninos", "el tono de la voz" o "no tener una actitud varonil". Una estudiante relata como acontecimiento habitual que en la cursada haya un chico "y que vayan todas a ese uno. Igual hay mucha homosexualidad también":

P- ¿Masculina?

R- Masculina si, femenina no tengo ni idea. La puerta de los baños, si querés ir a recabar información, en la puerta de los baños hay mucha homosexualidad femenina.

$\mathrm{P}$ - ¿Y los chicos sí se ven?

R- No, pero se nota. Se nota. "Prestame tu rímel", he escuchado alguna vez: "quiero que me recomiendes tu rímel". Se nota en amaneramientos, o también lo hacen explícito. Pero siempre decíamos que de diez hombres que hay en Psico siete son homosexuales. Tenemos que ir nosotras a ingeniería 
y está todo bárbaro. [Patricia. Entrevista realizada en noviembre de 2009 por el autor].

Así como circula esta representación recurrente respecto de los estudiantes varones, una referencia habitual versa sobre la estudiante de psicología, que constituye otra figura arquetípica: frágil emocionalmente, "habla de chicos", su aspecto es de "oficinista", "estudia para el parcial" e incluso suele abandonar la carrera, motivo por el cual, "se ven más varones en los últimos años de cursada". Sin embargo, las inscripciones a las puertas de los baños remiten, al menos en términos de visibilidad, a su opuesto: "Levanten las manos les y bi de Psico", "Pónganse un cartelito, me siento la única acá", “¿Dónde están las lesbianas en esta facultad?", son sólo algunas citas escritas en las puertas de los baños de mujeres, representativas de la necesidad de presentificar en el espacio de facultad a sujetos que, aunque no exclusivamente, permanecen invisibles: las mujeres lesbianas. Así, las escenas de intimidad pública son habitualmente heterosexuales, inscriptos en los guiones de género y sexualidades socialmente esperados (Gagnon, 2006): al ser pocos, algunos varones sostienen que existe la posibilidad de "pegar veinte minas", en la medida en que en las clases "hay para elegir", aunque desde el punto de vista de una entrevistada, "las chicas nos morimos de hambre" aunque "el [varón] que tiene actitud, gana".

El ordenamiento particular que los y las estudiantes, en este caso de Psicología, realizan respecto de las expresiones e identidades sexo-genéricas en el tiempo/ espacio universitario pone en relieve, en primer plano, una diferenciación en términos de mayoría/ minoría: mayoría de mujeres; minoría de varones; mayoría de varones gays; minoría de heterosexuales. Esta jerarquización, si en las narrativas tienen una dimensión considerable, su traducción en la sociabilidad, sin embargo, da cuenta de otra economía: "nadie ve parejas" del mismo sexo, afirma una estudiante de Psico, mientras que otra argumenta que "hay una cosa a pesar de todo como muy conservadora, ¿no? Acá se bancan al puto pero no se bancan a la parejita. Quieren la amiga loca, nada más". Como marca Pecheny, el problema mayor en un entorno heteronormativo no reside en "la dimensión puramente sexual de la identidad homosexual, sino en su expresión pública como afecto, amor o compromiso" y que se percibe en el cercenamiento "de algunos gestos cotidianos, por ejemplo caminar del brazo por la calle o de besarse en público" (Pecheny, 2002: 130). Efectivamente, si las expresiones y prácticas no heterosexuales no son censuradas ("es una elección, no está mal", dice un entrevistado;"yo diferencié qué es natural y qué no es. Como que estaba implícito que algo era natural. Quise decir habitual, que no se da con tanta habitualidad", se corrige una estudiante, respecto del modo en que se refirió en una primera entrevista a las relaciones entre mujeres) en cambio, sus posibilidades de visibilidad son, cuanto menos, más complejas. Mediante la denominación identidades discretas, Pecheny refiere al modo en que se estructura la sociabilidad de personas homosexuales a partir de diferen- 
tes estrategias en torno al gerenciamiento del secreto (Pecheny, 2002). Pero si el horizonte en ambas facultades es heteronormativo, la configuración tiempo, espacio, afectos diferente materializa, de un modo singular, esa normatividad y sus posibilidades de desestabilización.

En Exactas, si bien aparece para algunas carreras específicas la misma descripción respecto de Psico ("en Biología somos todas mujeres. Hay re poco varones y los que hay son gays"; "de hecho la mayoría de mis amigos de la carrera son gays") la forma de sociabilidad, como modalidad particular de estar juntos produce otra regulación. La figura del consorcio nombra no sólo una escala sino también un tipo de lazo centrado en la mirada atenta o el alto grado de conocimiento de estudiantes entre sí y con los y las docentes. Como correlato de su alta intensidad (referida en el mundo de las amistades, las posibilidades de realizar distintas actividades de formación y recreativas) produce también, en palabras de un estudiante de computación que se autoidentifica gay, una "presión" particular.

Poco frecuentes en Psico, algunas situaciones coloquiales de hostigamiento, en comentarios de fuerte contenido homofóbico, son referidas y generalmente impugnadas, en los relatos de estudiantes. Estas provenientes tanto de los pares como de docentes (como caso paradigmático por su magnitud son las declaraciones de un profesor, quien sostuvo públicamente en 2009, y ratificando los dichos realizados en la misma línea tiempo atrás: "Basta de hipocresía yo prefiero a mi hijo muerto que verlo conviviendo con otro hombre"). La presión debido al alto grado de interconexión, propicia prácticas de estigmatización y discriminación -como expone Jones- a partir de mecanismos como burlas, chismes, señalamientos, cargadas, insultos, entre otros, como un modo de disciplinar el comportamiento en el espacio público (Jones, 2010).

Sin embargo, esta misma presión (percibida y experimentada) constituye la base de desestabilización de esa normatividad. Una serie de acontecimientos personales ("uno de los chicos que era biólogo le dijo a su compañero que era gay, y resultó que el otro compañero también era gay"; ponerse de novio a partir de un intercambio en la biblioteca, "chapar en una fiesta"), en conflicto con los patrones heteronormativos referidos, habilita un tipo de reflexividad (sobre sí, sobre los otros, sobre la institución, y más allá) que da lugar a un tipo de sociabilidad organizada (Gurvitch, 1941). "Diversidad Sexual en Exactas y Naturales" (inicialmente se denominó "Comunidad Homosexual de Exactas y Naturales") se configura en este sentido como un grupo de estudiantes que se constituye con la idea de hacer "algo específicamente político sobre la diversidad sexual y los derechos". Entre otras actividades, realizaron durante su inicio ciclos de cine sobre diversidad sexual en el aula magna, convocaron a la $18^{\circ}$ Marcha del Orgullo LGBT y realizaron intervenciones en el espacio de la facultad. Como afirma un estudiante que participa en ese espacio: 
el hecho es que se ponga un cartel, se arme un nombre, y se haga una mínima actividad, le va a dar a un montón de gente la sensación de que no están solos. Eso fue el objetivo principal, sentimos eso de que aunque quizás de afuera parece que está todo bien con los gays, la realidad es que hay mucha gente que no sale del closet. [Diego. Entrevista realizada en julio de 2010 por el autor].

Agruparse tuvo por objetivo "descomprimir"; en otros términos, desestabilizar un tipo de regulación sexual operante en su continuum con el afuera de las fronteras de la universidad ("No podemos decirle a cualquiera que somos gays, por una cuestión de que uno viene de la calle, de la casa, de la Argentina y te da miedo", dice el mismo entrevistado).

Lo que en primera persona es expresado como "descomprimir", es descripto por otros y otras estudiantes de Exactas como un rasgo más o menos habitual del mapa de relaciones propio de la vida universitaria, en el que impera respecto de las sexualidades no heterosexuales el "todo bien", o a lo sumo, el "como en todos lados". Ese espacio constituye -de un tiempo a esta parte- un territorio en el que es común "ver gente del mismo sexo besándose por los pasillos del Pabellón II" (el edificio más grande que posee Exactas) en un espectro más amplio de relaciones eróticas y afectivas, en el que los límites físicos de la visibilidad se encuentran en redefinición por el trazado de nuevos límites políticos, arbitrarios y terreno de disputa.

\section{A modo de cierre}

La distinción entre itinerarios estudiantiles autogestionados e institucionalizados refiere, de un modo esquemático, a dos modalidades que adquiere la vida con otros/as según una constelación específica entre prescripciones institucionales, condiciones de experiencia y una trama intersubjetiva particular, y que es posible ilustrar a partir de las figuras de la ciudad y el consorcio. La figura de la ciudad, para referir a Psico, condensa las características de anonimato y masividad que configuran un tipo de sociabilidad (de proximidad con otros/as) menos intensa, con excepción de las relaciones de amistad que -vale para ambas facultades- dan cuenta de un vínculo de comunión delimitado en la zona común de las afinidades y elecciones. En la dimensión regulatoria en torno a las sexualidades, la primacía de la heterosexualidad se traduce en un horizonte posible de escenas de intimidad pública. En los lazos eróticos y afectivos no heteronormativos, la autogestión radica en la experiencia, nunca "del todo" personal (Scott, 2000) de control de la propia biografía, que produce un tipo de sociabilidad discreta, más o menos exenta de situaciones de hostigamiento explícito en el espacio público, pero al mismo tiempo privado del espacio público.

En Exactas, en cambio, prevalece un tipo de sociabilidad comunitaria, en contraste con la experiencia del anonimato, debido a la intensidad en el cono- 
cimiento de otro/as. Acerca de la dimensión normativa de las sexualidades en ese espacio vale un último comentario respecto de la vergüenza, como locus problemático que puede orientar futuras indagaciones.

El investigador peruano Giancarlo Cornejo Salinas, desde una perspectiva queer, sostiene que la privatización de la sexualidad no heterosexual implanta la vergüenza en toda subjetividad de quienes se reconocen alejados de la heteronormatividad; de ahí que una condición para transgredirla sea aceptar la vergüenza y encarnarla (Cornejo Salinas, 2011). La sociabilidad de tipo institucionalizada (o mediada institucionalmente) de los y las estudiantes de Exactas habilita, pese a los signos manifiestos de hostilidad respecto a las expresiones e identidades no heterosexuales, modos de organización que cuestionan críticamente el espacio universitario como territorio heteronormativo. La mayor intensidad de los vínculos es referida como una situación de gran presión. Sin embargo, desde una mirada comparada con otra facultad (Psico), es posible relativizar esta creencia (no así la vivencia de cada uno). A diferencia de la sociabilidad desplegada en un universo anónimo que repliega la gestión de la vida afectiva y sexual a las estrategias individuales -"la masa es aquel que exige las explicaciones más detalladas", sostiene Gurvitch (1941: 44) - la sociabilidad intensa posibilita una estrategia colectiva que permite encarnar en el espacio público aquello que produce vergüenza. De ahí la paradoja de que el consorcio pueda ser un espacio de libertad, y la gran ciudad un infierno grande.

En su conjunto, el análisis se de estos procesos -que denomino como regulaciones sexo- genéricas- se dirigen a afirmar al menos dos cuestiones. En primer lugar, a visibilizar la gestión que los y las estudiantes realizan cotidianamente de su expresión e identidad de género y sexualidad en función de los implícitos institucionales que estructuran los modos de habitar la universidad. En segundo lugar, se busca señalar no sólo que la universidad constituye un espacio heteronormativo sino también que es un territorio que ofrece, en las singularidades que adquieren las experiencias de los sujetos, posibilidades de desestabilización de esta normatividad.

\section{Referencias Bibliográficas}

- $\quad$ Arfuch, L. (2005). Cronotopías de la intimidad. En Arfuch L. (comp.) Pensar este tiempo. Espacios, afectos, pertenencias. Buenos Aires: Paidós.

- $\quad$ Bachelard, G. (1975). La poética del espacio. México: Fondo de Cultura Económica.

- Barthes, R. (2002). Fragmentos de un discurso amoroso. Buenos Aires: Siglo XXI.

- Blanco, R. (2010). La universidad opaca. Vida cotidiana, normatividad y sexualidades desde las experiencias de estudiantes. Revista Ciencias Sociales, 76, 62-63.

- Carli, S. (2006). Figuras de la amistad en tiempos de crisis: la universidad pública y la sociabilidad estudiantil. En Diker G. y Frigerio G., Educar: figuras y efectos del amor, Buenos Aires: Del estante. 
- Censo estudiantes 2004. Universidad de Buenos Aires, Secretaría de Asuntos Académicos.

- Cornejo Salinas, G. (2011). La guerra declarada contra el niño afeminado: Una autoetnografía 'queer'. En Íconos. Revista de Ciencias Sociale, 39. Quito: Facultad Latinoamericana de Ciencias Sociales.

- De Certeau, M. (1996). La invención de lo cotidiano I. Artes de hacer. México DF: Universidad Iberoamericana.

- Gagnon, J. (2006). O uso explícito e implícito da perspectiva da roteirização nas pesquisas sobre sexualidade. En J. Gagnon, Uma interpretação do desejo : ensaios sobre o estudo da sexualidade, Río de Janeiro: Garamond.

- Guber, R. (1991). El salvaje metropolitano. A la vuelta de la antropología postmoderna. Reconstrucción del conocimiento social en el trabajo de campo. Buenos Aires: Legasa.

- $\quad$ Gurvitch, G. (1941). Las formas de la sociabilidad. Buenos Aires: Losada.

- Jones, D. (2010). Sexualidades adolescentes. Amor, placer y control en la Argentina contemporánea. Buenos Aires: CICCUS/CLACSO.

- Margulis, M. y otros (2007). Familia, hábitat y sexualidad en Buenos Aires. Investigaciones desde la dimensión cultural. Buenos Aires: Biblos.

- Moreno, A. (2008). La invisibilidad como injusticia. Estrategias del movimiento de la diversidad sexual. En Pecheny, Figari y Jones (comps.), Todo Sexo es Político. Estudios sobre sexualidades en Argentina. Buenos Aires: El Zorzal.

- $\quad$ Pecheny, M. (2008). Introducción. Investigar sobre sujetos sexuales. En Pecheny, Figari y Jones (comps.), Todo Sexo es Político. Estudios sobre sexualidades en Argentina. Buenos Aires: El Zorzal.

- (2002), Identidades discretas. En Arfuch L. (comp.), Identidades, sujetos y subjetividades. Buenos Aires: Prometeo.

- Rubin, G. (1989). Reflexionando sobre el sexo: notas para una teoría radical de la sexualidad. En Vance C. (comp.) Placer y peligro. Explorando la sexualidad femenina. Madrid: Revolución.

- Simmel, G. (2002). Cuestiones fundamentales de sociología. Buenos Aires: Gedisa.

- Scott, J. (2000) "El género: una categoría útil para el análisis histórico", en Lamas, M. (comp.) El género. La construcción cultural de la diferencia sexual, México, PUEG. 